\title{
COVID-19 guidance in humanitarian settings: The need for dynamic guidelines adapted to changing humanitarian crisis contexts
}

\section{Alex Odlum ( $\sim$ alex.odlum@graduateinstitute.ch )}

Graduate Institute of International and Development Studies: Institut de Hautes Etudes Internationales et du Developpement https://orcid.org/0000-0002-9281-9835

\section{Rosemary James}

University of Geneva Faculty of Medicine: Universite de Geneve Faculte de Medecine

\section{Audrey Mahieu}

Université de Genève: Universite de Geneve

\section{Karl Blanchet}

Université de Genève: Universite de Geneve

\section{Chiara Altare}

Johns Hopkins University

\section{Neha Singh}

London School of Hygiene \& Tropical Medicine

\section{Paul Spiegel}

Johns Hopkins University

\section{Research Article}

Keywords: COVID-19, Humanitarian, Evidence, Guidance, Online platform

Posted Date: June 17th, 2021

DOI: https://doi.org/10.21203/rs.3.rs-625232/v1

License: (c) (1) This work is licensed under a Creative Commons Attribution 4.0 International License. Read Full License 


\section{Abstract}

Background: For humanitarian organisations to respond effectively to complex crises, they require access to up-to-date evidence-based guidance. In addition, the COVID-19 crisis has highlighted the importance of adapting and updating global guidance to context-specific and evolving needs in fragile and humanitarian settings. Our study aimed to understand the use of evidence in humanitarian responses during COVID-19.

Methods: We collected and analysed COVID-19 guidance documents, and conducted semi-structured interviews remotely with a variety of humanitarian organisations responding and adapting to the COVID19 pandemic. We used the COVID-19 Humanitarian platform, a website established by three universities in March 2020, to solicit, collate and document these experiences and knowledge.

Results: We collected 180 guidance documents, and after excluding those that did not meet our inclusion criteria, analysed 131. We conducted 80 interviews with humanitarian organisations in the field, generating 61 published field experiences. Although COVID-19 guidance was quickly developed and disseminated in the initial phases of the crisis (from January to May 2020), updates or ongoing revision of the guidance has been limited. Interviews conducted between April and September 2020 showed that humanitarian organisations have responded to COVID-19 in innovative and context-specific ways, but have often had to adapt existing guidance to inform their operations in complex humanitarian settings.

Conclusion: Experiences from the field indicate that adopting guidance to respond and adapt to COVID-19 is a complex process requiring innovation and collaboration based on the local context and resource availability. Global guidance aimed at humanitarian actors could be improved through responsive incorporation of contextualised field experiences in a timely manner using real-time feedback loops through online platforms like the COVID-19 Humanitarian platform.

\section{Background}

Humanitarian crises affect diverse peoples in a wide range of contexts around the world, with an estimated 168 million people needing assistance and protection in 2020 [1]. For humanitarian organisations to respond effectively to complex crises, they require access to up-to-date evidence-based guidance detailing which interventions are recommended and which adaptations can be effective in certain contexts or circumstances. The need for more evidence-informed humanitarian response is wellestablished [see e.g. ,2-4]. More recently, the COVID-19 crisis has highlighted the importance of adapting and updating global guidance to meet "context-specific and evolving needs in fragile settings" [5].

Requests from humanitarian actors to some of the authors for technical advice on how to respond to the COVID-19 pandemic in humanitarian settings highlighted a gap in the existing evidence base, as well as a need for real-time feedback loops to relay grounded experience and field innovations to global standard setters to ensure timely and relevant guidance. To help meet these requests, three universities joined forces to develop the COVID-19 Humanitarian platform 
(https://www.covid19humanitarian.com/). Between March and August 2020, we reviewed 180 global level guidance documents. By December 2020, we had documented 61 field experiences shared by humanitarian organisations across three main domains: preparing for and responding to COVID-19, adapting existing interventions to COVID-19, and cross-cutting issues relevant to humanitarian settings [6]. In addition, our team and partners facilitated weekly webinars on emerging and controversial issues attended by both public health experts and humanitarian practitioners working in crisis-affected countries (https://www.ready-initiative.org/webinars/).

Review and analysis of the guidance documents alongside qualitative data from field experiences collected through the COVID-19 Humanitarian platform point towards two trends. First, although COVID19 guidance was quickly developed and disseminated in the initial phases of the pandemic (from approximately January to May 2020), updates or ongoing revision of the guidance has been limited. Second, field experiences collected from April 2020 to September 2020 show that humanitarian organisations have responded to COVID-19 in innovative and context-specific ways, but have often had to adapt global guidance to inform their operations in complex humanitarian settings.

We argue that these trends reflect complexities in the way guidance is used and implemented by humanitarian organisations, and indicate the absence of adequate feedback loops for responsively reintegrating humanitarian field experience into global guidance and standard setting processes. Joining up these loops could improve the quality and use of evidence in current and future humanitarian response. In this paper, we review existing guidance documents for COVID-19, assess their relevance for humanitarian settings, and analyse their use by humanitarian actors in documented field experiences.

\section{Methodology}

\section{Phases}

Research for the COVID-19 Humanitarian platform evolved through four main phases. In the first phase (March 2020), we established research teams at the the three universities, designed an analytical framework (Figure 1), and set up the COVID-19 Humanitarian platform to collect and share guidance documents and field experiences for each section and area of the framework.

The second phase (April to September 2020) involved the definition of standard operating procedures to collect guidance and gather field experiences, real-time collection of guidance documents, inductive learning from weekly webinars, and a series of qualitative interviews with humanitarian organisations on their responses and adaptations to COVID-19. We searched for guidance documents, assessed them following pre-established criteria, and posted relevant documents on the platform. In addition, we collected, reviewed and summarised field experiences into a standard format for the online platform. We actively collected field experience data until September 2020, and posted some additional experiences until December 2020. The platform remains open to spontaneous submissions from humanitarian organisations, and continues to host the data for reference. 
Summary analysis of guidance documents and field experience data marked the third phase (August to September 2020). This analysis identified general trends about the nature of guidance documents and field experiences we had collected.

Reflection on these trends generated supplementary research questions for further analysis, which marked the fourth phase. These questions were:

1. What guidance exists for responding to COVID-19 in humanitarian settings?

2. To what extent is this guidance specific to humanitarian contexts?

3. How have humanitarian organisations followed or departed from COVID-19 guidance in practice?

4. How has COVID-19 guidance evolved or been updated since March-May 2020 ?

5. How can guidance setters better integrate innovations and lessons from rapidly evolving field experience during future humanitarian health crises?

\section{Collection and analysis of guidance documents}

To identify available COVID-19 guidance documents, we actively prospected major humanitarian agencies' websites and portals: United Nations (UN) agencies and the Inter-Agency Standing Committee (IASC), Centers for Disease Control and Prevention (CDC), The International Red Cross and Red Crescent Movement, global humanitarian clusters, international non-governmental organisations (INGOs), Reliefweb, and the Active Learning Network for Accountability and Performance in Humanitarian Action (ALNAP). In addition, we contacted humanitarian organisations to collect guidance on COVID-19. The guidance documents were individually assessed and we met on a weekly basis to discuss and validate the guidance that met the criteria to be included on the COVID-19 Humanitarian Platform. Selection criteria required the guidance to be:

- Evidence-based (or based on solid field-experience),

- Unbiased (e.g. not for promotional purposes),

- Applicable to low income or humanitarian settings, and

- Actionable.

We then analysed the identified guidance documents using Microsoft Excel and Statistical Package for the Social Sciences (SPSS) to cross tabulate the number of documents per framework area.

\section{Field experience interviews}

\section{Sampling}

We solicited field experiences from humanitarian organisations via a form on the online COVID-19 Humanitarian platform, as well as through outreach across our research team's own networks of practitioner contacts. Eligible interview participants were any employee of a humanitarian organisation that was responding or adapting interventions to COVID-19, who had sufficient operational knowledge of 
the intervention to describe it in detail. Participants based in country (national or sub-national levels) were preferred, but headquarter or regional level participants were also included if they had detailed knowledge of the context they were describing. To mitigate selection bias, we tracked the geographic coverage of the field experiences collected, aiming to collect at least one experience from each of the humanitarian crises listed in United Nations Office for the Coordination of Humanitarian Affairs (UN OCHA's) Global COVID-19 Response Plan [7], as well as the top five refugee hosting countries by number of refugees [8]. Similarly, we aimed to cover all sections and areas of the analytical framework in Figure 1, to ensure data on a wide range of humanitarian interventions and techniques, and public health issues. Finally, the type of organisations interviewed naturally varied, with experience collected from UN agencies, major INGOs, government agencies, non-governmental organisations, and local civil society and grass-roots initiatives. Overall, the location, intervention and organisation types consulted were sufficiently diverse to generate a spectrum of experiences at different levels of humanitarian operations.

\section{Data collection}

The main data collection approach involved emailing contacts and representatives of humanitarian organisations to request their participation in a 30- to 60-minute interview about their experience implementing or adapting programs to COVID-19. Each interview aimed to focus on an area of the framework in Figure 1 as specifically as possible: most organisations have responded to COVID-19 with multifaceted interventions, but we aimed to extract sufficient detail on a specific intervention by focusing on one key area at the lowest possible level of the framework. These were most frequently risk communication ( $n=8$ interviews), triage $(n=7)$, context analysis $(n=6)$, food security, particularly food, cash and voucher distributions $(n=6)$, nutrition $(n=5)$, infection prevention and control $(n=5)$, mental health and psychosocial support $(n=5)$, education $(n=3)$, and sexual and gender-based violence (SGBV) $(n=3)$. Lebanon, Pakistan, South Sudan, Afghanistan, Democratic Republic of the Congo (DRC), Bangladesh, Haiti, Libya, Nigeria, and Greece were the most frequently covered countries.

Each interview followed a semi-structured guide with prompts to assist enumerators extract more detailed information from participants. After conducting the interviews, we cleaned notes and transcripts and drafted summaries according to standardised templates that covered context, intervention and rationale for adaptation, process modalities, challenges and enabling factors, and related documents.

Each field experience summary underwent a two-stage review process: firstly, a panel of experts from each of the three universities involved in the COVID-19 Humanitarian project reviewed the field experience summary; secondly, we sent the finalised summary to the concerned organisation for review and final approval to publish. We believe the interviews comply with the "Consolidated criteria for reporting qualitative research checklist" [9].

\section{Data analysis}

We conducted structured qualitative content analysis of the field experience interviews using NVivo Version 12 Pro software. We considered the final published field experience as the main data source, as 
these were clean and standardised allowing for the most comparable interpretations across experiences. Where clarity or further detail was needed, we referred back to the interview recordings, transcripts or notes. After initial familiarisation of the data, we combined deductive and inductive manual coding in parallel to analyse specific questions about the usage and usefulness of COVID-19 guidance by humanitarian organisations. At a second stage, we checked for further references to guidance by querying all text for a long list of synonyms and related words to "guidance", including: guidelines, recommendations, advice, standards, and protocols (see Table 4). Each field experience represented a unique case, and the analysts deductively coded each case summary according to the following node structure:

- Whether the organisation referred to guidance explicitly or implicitly,

- Explicit $=$ mentioned specific guidance source or guidance in general

- Implicit = guidance not mentioned but can be implied from the context.

- Whether the organisation used the guidance directly, indirectly, or not at all,

- Direct = using, referring to, basing implementation on guidance, standards, principles, recommendations.

- Indirect = reading, consulting, hearing about guidance, standards, principles, recommendations

- Rejected = not using, referring to, basing implementation on guidance, standards, principles, recommendations.

- Whether the organisation evaluated the guidance positively, negatively or missing.

- Positive = useful, helpful, relevant or accurate

- Negative $=$ not useful, unhelpful, irrelevant or inaccurate .

- Missing = would have been helpful but was unavailable, missing or lacking.

Following the structured, deductive coding, we applied a latent content analysis technique, using a constructivist approach to identify further themes in the data.

\section{Results}

\section{Guidance documents}

In total, we identified and reviewed 180 guidance documents. This analysis considers 131 documents, with 49 guidance documents excluded from the analysis for the following reasons stated in Fig. 2.

Out of 180 guidance documents identified and reviewed, our weekly review panel discarded 13 because they did not meet the selection criteria: they either were not evidence-based, not actionable, biased, or did not apply to either low-income or humanitarian settings. Sixteen guidance documents were duplications (the same document found by different researchers) and three were already included in the dataset in another language. Nine guidance documents were published before 2020 (some were published in 2017 
or 2019) and did not specifically address COVID-19. They were either guidance for past epidemics or generic guidance on Risk Communication and Community Engagement (RCCE). Four were not guidance documents but links to other guidance gathering websites. The copies of three guidance documents listed in the dataset could not be found at the analysis stage neither in the web-based platform, nor in the web typing their title in the search engine.

Figure 3 shows the number of guidance documents published or co-published per type of organisation. In total, 64 unique organisations published 131 documents. Some organisations published multiple documents, and some co-publications involved multiple organisation types. This resulted in 171 publishing organisations. Out of the 131 guidance documents included for analysis, 31 (24\%) were published or co-published by World Health Organization (WHO), 20 (15\%) by United Nations Children's Fund (UNICEF), 10 (8\%) by IASC, 9 (7\%) by United Nations High Commissioner for Refugees, 6 (5\%) by World Food Programme, and 6 (5\%) by United Nations Population Fund. A wide range of organisations produced five or fewer of the guidance documents.

In terms of relevance, out of the 131 guidance documents, 55 (42.1\%) were relevant to all settings, 39 $(30 \%)$ were relevant to low and middle-income countries, two (1.5\%) were relevant to low-income countries and $35(27 \%)$ were relevant to humanitarian settings. All 131 guidance documents included were available in English. Only 37 (28\%) also existed in French and 19 (14\%) in Arabic, despite these being common languages in many humanitarian settings.

We identified relevant guidance published between the 30 January 2020 and 31 May of 2020, although most originated between mid-March and end of April 2020. As of August 2020, only 24 (18,3\%) of the 131 guidance documents had been updated since their first publication, despite the swiftly evolving knowledge and evidence-base on the nature of SARS-COV-2 and its impacts, as well as continually evolving lessons on effective public health and humanitarian response modalities to the COVID-19 pandemic (Fig. 4).Further cross tabulation analysis showed that the WHO and the CDC were among the first organisations to publish guidance documents. Although this guidance did not specifically target lowincome and humanitarian settings, we deemed it globally relevant. Guidance from the Africa CDC, by contrast, was not published until later in April 2020.

Ultimately, the guidance documents covered a wide-range of the COVID-19 framework areas, as shown in Table 1. 
Table 1

Number and percentage of guidance documents per framework area

\begin{tabular}{|c|c|c|c|c|}
\hline Category & Sub-category & Area & $\begin{array}{l}\text { Number } \\
\text { (n) }\end{array}$ & $\begin{array}{l}\text { Percentage } \\
(\%)\end{array}$ \\
\hline \multirow{15}{*}{$\begin{array}{l}\text { COVID- } \\
\text { Specific }\end{array}$} & \multirow[t]{5}{*}{ Case Management } & Management of mild cases & 3 & 2.3 \\
\hline & & $\begin{array}{l}\text { Management of severe cases } \\
\text { (non-Intensive Care) }\end{array}$ & 0 & 0 \\
\hline & & $\begin{array}{l}\text { Management of severe cases } \\
\text { (Intensive Care) }\end{array}$ & 2 & 1.5 \\
\hline & & Triage & 3 & 2.3 \\
\hline & & $\mathrm{N} / \mathrm{A}$ & 3 & 4.6 \\
\hline & \multirow{2}{*}{$\begin{array}{l}\text { Investigation \& } \\
\text { surveillance }\end{array}$} & Surveillance & 4 & 3.1 \\
\hline & & Investigation & 0 & 0 \\
\hline & \multirow[t]{5}{*}{ Prevention } & Infection Prevention \& Control & 5 & 3.8 \\
\hline & & Isolation Measures & 2 & 1.5 \\
\hline & & $\begin{array}{l}\text { Risk Communication and } \\
\text { Community Engagement }\end{array}$ & 10 & 7.6 \\
\hline & & Rumour management & 1 & 0.8 \\
\hline & & $\mathrm{N} / \mathrm{A}$ & 2 & 1.5 \\
\hline & \multirow{2}{*}{$\begin{array}{l}\text { Risk-assessment and } \\
\text { context analysis }\end{array}$} & Context analysis & 0 & 0 \\
\hline & & Health Facility Assessment & 1 & 0.8 \\
\hline & $\mathrm{N} / \mathrm{A}$ & $\mathrm{N} / \mathrm{A}$ & 6 & 4.6 \\
\hline \multirow{9}{*}{$\begin{array}{l}\text { COVID- } \\
\text { Sensitive }\end{array}$} & \multirow[t]{7}{*}{ Essential Health Services } & Communicable diseases & 5 & 3.8 \\
\hline & & Injury and Rehabilitation & 0 & 0 \\
\hline & & $\begin{array}{l}\text { Maternal, Newborn and Child } \\
\text { Health }\end{array}$ & 5 & 3.8 \\
\hline & & $\begin{array}{l}\text { Mental Health and Psychosocial } \\
\text { Support }\end{array}$ & 3 & 2.3 \\
\hline & & Non-communicable diseases & 1 & 0.8 \\
\hline & & Sexual and Reproductive Health & 1 & 0.8 \\
\hline & & $\mathrm{N} / \mathrm{A}$ & 1 & 0.8 \\
\hline & Human Resources & $\mathrm{N} / \mathrm{A}$ & 2 & 1.5 \\
\hline & Other programmatic & Education & 5 & 3.8 \\
\hline
\end{tabular}




\begin{tabular}{|c|c|c|c|c|}
\hline Category & Ŝruas & Area & $\begin{array}{l}\text { Number } \\
\text { (n) }\end{array}$ & $\begin{array}{l}\text { Percentage } \\
(\%)\end{array}$ \\
\hline & & Food security and livelihoods & 8 & 6.1 \\
\hline & & Nutrition & 7 & 5.3 \\
\hline & & $\begin{array}{l}\text { Water, Sanitation and Hygiene } \\
\text { (WASH) }\end{array}$ & 5 & 3.8 \\
\hline & Prioritisation & $\mathrm{N} / \mathrm{A}$ & 4 & 3.1 \\
\hline & N/A & $\mathrm{N} / \mathrm{A}$ & 3 & 2.3 \\
\hline \multirow{11}{*}{$\begin{array}{l}\text { Cross- } \\
\text { cutting }\end{array}$} & Coordination & $\mathrm{N} / \mathrm{A}$ & 2 & 1.5 \\
\hline & Ethics & $\mathrm{N} / \mathrm{A}$ & 3 & 2.3 \\
\hline & Gender & $\mathrm{N} / \mathrm{A}$ & 7 & 5.3 \\
\hline & Logistics & $\mathrm{N} / \mathrm{A}$ & 0 & 0 \\
\hline & Monitoring \& Evaluation & $\mathrm{N} / \mathrm{A}$ & 2 & 1.5 \\
\hline & \multirow[t]{4}{*}{ Protection } & Attacks on healthcare & 0 & 0 \\
\hline & & Diversity and inclusion & 6 & 4.6 \\
\hline & & General protection & 12 & 9.2 \\
\hline & & $\begin{array}{l}\text { Sexual and Gender based } \\
\text { violence }\end{array}$ & 6 & 4.6 \\
\hline & \multirow[t]{2}{*}{ N/A } & $\mathrm{N} / \mathrm{A}$ & 1 & 0.8 \\
\hline & & Total & 131 & 100 \\
\hline
\end{tabular}

Note: N/A means that the guidance covered a wide range of categories. For instance, some COVIDspecific guidance documents included sections on case management, investigation \& surveillance, prevention and risk assessment \& context analysis.

\section{Field experiences}

Between April and December 2020, the team conducted 80 qualitative interviews with humanitarian workers from local civil society and grassroots initiatives, international and national NGOs and UN agencies based primarily in the field. 
Table 2

Number of submissions to COVID-19 Humanitarian

platform per status

\begin{tabular}{|ll|}
\hline Submission status & Number $(\mathbf{n})$ \\
\hline Submissions proceeding to interview & 12 \\
\hline Submissions excluded before interview & 2 \\
\hline Total submissions & $\mathbf{1 4}$ \\
\hline
\end{tabular}

This process generated 61 field experience summaries covering 40 countries that underwent a standardised review by the three universities before being uploaded to the COVID-19 Humanitarian platform. In total, 14 organisations submitted their experiences via the online form (Table 2). However, these submissions were not all written in a way that met the standards of clarity and detail for the published field experiences, and so they required curation. Two submissions were clearly opportunistic marketing or spam, and therefore ignored. For the 12 genuine but incomplete submissions, we carried out follow up interviews, which brought the data collection process into line with the main approach of conducting with interviews with the contacts of our research team and snowballed participants.

Table 3

Number of field experience interviews per outcome of interview process

\begin{tabular}{|ll|}
\hline Outcome of interview process & Number (n) \\
\hline Published summary & 61 \\
\hline Excluded after interview (insufficient information) & 8 \\
\hline Excluded after drafting summary (panel deemed unsuitable) & 3 \\
\hline Process incomplete/ongoing (at time of writing) & 8 \\
\hline Total interviews & $\mathbf{8 0}$ \\
\hline
\end{tabular}

We excluded eight experiences after the interviews for lacking sufficient data (Table 3). In these cases, the initial information collected was too general or incomplete, and pursuing further details would not have been feasible or too costly. For example, if after prodding or follow-up, the interviewee revealed no more specific or detailed information than what could be found on the organisation's website or other published communication materials about their activities, we dropped the process. Panel reviewers also found three interventions to be irrelevant, controversial, or unsuitable for further promotion, even in light of their specific context. We decided to exclude these data after deliberation and based on consensus. Eight interviews could not be converted to published summaries due to researcher unavailability or 
remained in process at the time of analysis and writing. Singh et al. [10] summarised in more detail the field experiences collected for the COVID-19 Humanitarian platform.

\section{References to guidance in the field experiences}

The field experience interviews focused on soliciting detailed descriptions of the adaptations and interventions humanitarian organisations had made or were making to continue operating in the context of the COVID-19 pandemic. Analysing the summarised field experiences, we found multiple instances where organisations explicitly mentioned guidance documents (such as "WHO guidelines for personal protective equipment") when describing their interventions and adaptions to COVID-19. In addition, we found cases where humanitarian organisations made implicit references to guidance documents, for example when a participant explained the way staff followed or departed from "recommended" procedures. In these references to guidance, we identified three major themes: organisations refer to authoritative global or international guidance and adapt it to context; organisations refer to guidance disseminated from headquarter levels and adapt it to context; organisations combine various sources of guidance and adapt it to context. While our sampling strategy did not allow us draw conclusions about which approach is more frequent among humanitarian organisations, the following examples demonstrate the variety of ways guidance is used and adapted.

\section{Adoption and adaptation of global guidance}

Humanitarian organisations that explicitly mentioned guidance documents when explaining their COVID19 responses and adaptations often referred to documents issued by WHO and the IASC, two predominant global bodies for public health and international humanitarian response respectively. In particular, respondents referenced WHO guidance on a variety of topics, including case management, personal protective equipment (PPE), case definitions, RCCE, infection prevention and control (IPC), screening, and psychosocial support (PSS). For example, War Child's psychosocial support interventions were adapted based on the Lebanon's Ministry of Health $(\mathrm{MoH})$, WHO, and IASC guidelines and from the local coordination group headed by UNICEF. Organisations also specifically mentioned global guidance developed by academic institutions, such as the London School of Hygiene and Tropical Medicine (LSHTM) guidance for preventing COVID-19 infections among high-risk individual in camps and camplike settings [11].

In addition to citing guidance documents, humanitarian organisations explicitly referred to other communications materials issued by authoritative humanitarian and public health agencies like WHO and IASC. The also explicitly referred to materials issued by local and national governments, such as lists

of frequently asked questions and answers (FAQs), posters or leaflets, and social media material with key messages. An example of this was explained by the Mixed Migration Centre, who drew on FAQs from WHO (https://www.who.int/covid-19) to verbally share information on COVID-19 with respondents after conducting phone interviews to collect data on migration trends. 
References to global guidance were not limited to documents and key message materials, but also covered other types of global standards and resources, including methodologies, models or indices. For example, in DRC, REACH worked with UN OCHA and a core group of information management actors to rapidly develop an index for measuring the level of vulnerability of each health zone during COVID-19, and the likelihood of negative impacts of the epidemic at this level. To do so, they referred to existing global vulnerability models, namely the European Commission's Index for Risk Management (INFORM) severity index [12].

Common across these references to international or global guidance was an additional explanation of how guidance was adapted, amended, translated, or otherwise contextualised. For example, an anonymous organisation in Iraq who described their health facility triage system explained how their volunteer distributed leaflets about COVID-19 were designed based on WHO guidelines, and translated by the organisation into Arabic. In this same case, the organisation also explained how they had referred to WHO guidelines for PPE, but had to ration usage of PPE because supply and stock lines were limited, and thus could not fully align their implementation with the guidance.

\section{Dissemination of headquarter level guidance}

In multiple cases, organisations at national and local levels adapted or generated guidance with assistance from their global headquarters. The International Rescue Committee's (IRC) shift to remote delivery of essential health services during a strict urban lockdown in Jordan involved close collaboration between the country office and headquarters. Firstly, technical documents from the IRC headquarters aided country intervention teams to develop their remote service delivery plans for primary healthcare and reproductive health clinics. Secondly, experts well-versed in this content immediately trained the teams on these new protocols via a webinar. As such, the rapid design of protocols and staff training allowed for an adaptation of services with only one week of interruption.

In a similar case, the Libyan Red Crescent Society (LRCS) received support from the International Federation of Red Cross and Red Crescent Societies (IFRC) to develop an appropriate COVID-19 RCCE strategy and virtual training for volunteers due to travel limitations. A respondent from LRCS explained this process:

We cannot go to Libya, they cannot come to us...there was no opportunity for us even to gather together with regards to the content [development]. Usually the content is developed by Geneva. The IFRC headquarters in Geneva, the healthcare units, the disaster and crisis unit, they developed the risk communication and community engagement guidelines. And this rapid training. And then we disseminate. So it goes to regional office, regional office disseminates to country offices and then we take it from there with a national title.

-The Libyan Red Crescent Society on the development of guidance and rapid trainingThe full field experience is reported here: https://www.covid19humanitarian.com/field_experience/? id $=112$ 
Another example of top-down dissemination from headquarters was the COVID-19 preparedness and response activities in Médecins Sans Frontières (MSF's) field hospital in Cox's Bazaar, Bangladesh. In this case, the field hospital used guidelines on disinfection and sterilisation, as well as on prone position (for the management of severe cases), which had been generated by MSF headquarters based on WHO guidance. In a similar way, IRC's mental health and psychosocial support (MHPSS) programming in Tanzania utilised IRC's global COVID-19 MHPSS operational guidance [13] to define key areas for adaptation. A similar strategy did not directly stem from headquarter guidance on COVID-19, but drew on past organisational experience to inform current response and adaptation. For example, MSF in Central African Republic (CAR) quickly adapted their existing PPE guidance based on previous experience from Ebola and Cholera outbreaks and in consultation with IPC experts.

\section{Combination of multiple guidance sources}

Humanitarian organisations also combined multiple sources of guidance to create procedures fit for the context in which they were operating, including language, culture, and resource-specific considerations. For instance, in describing their work on sensitising communities on COVID-19 and advocating for the protection of women from SGBV during the pandemic, Action Aid Haiti explained how they referred to guidance from WHO, the Haitian $\mathrm{MoH}$ and Action Aid International, but adapted these documents to the local context. Similarly, in South Sudan, Islamic Relief mentioned that it had been useful to adapt guidance from WHO, national and local authorities, to the local context to inform their health surveillance operations in porous border areas near Kenya. Doctors with Africa (CUAMM)'s operations in South Sudan also described needing to adapt their organisation's general guidelines for triage into a simplified version suitable for the specific context of a local health facility.

A variation on the approach of combining global with national and local guidelines was to refer to domestic guidelines designed for a foreign context in the context at hand. Medical Volunteers International, an INGO which operates health clinics in Greece, filled specific clinical gaps with foreign guidance issued from the UK's National Institute for Health and Care Excellence (NICE). The organisation deemed the NICE infection prevention and control guidance more appropriate than material issued by the Hellenic National Public Health Organisation, which although context-specific to Greece, targeted the public rather than the specialised health clinic settings where they operated and for which they needed guidance.

\section{Additional references to guidance}

During our analysis, we recognised that the word "guidance", or related stemmed words such as guide and guideline, did not cover the full spectrum of ways in which humanitarian organisations consider evidence when explaining their experiences responding and adapting to COVID-19. To address potential gaps in our analysis, we ran a query on the published field experience texts to identify synonyms and stemmed words that would also indicate reference to and usage of guidance by humanitarian organisations. This allowed us to gain more and deeper insights on the extent to which humanitarian 
responses and adaptations to COVID-19 were evidence-based, as well as the nature of evidence and guidance usage in programme design.

We first identified a long list of 40 synonyms for guidance and guidelines, looking for relevant terms in publicly available web-based dictionaries and thesauri (e.g., www.thesaurus.com). After running the long query list, we dropped 19 terms that returned no relevant matches or where the usage of the term did not imply reliance on, following or use of guidance. For example, if the example showed the organisation provided "guidance" to beneficiaries on handwashing, we excluded it. If, however, it implied the organisation followed guidance (or its synonyms) on handwashing, we included it. In total we identified, 85 examples for 21 terms.

Table 4 presents a selected example for each search term. While these are not directly transcribed quotes from organisations (they are extracted from the editorialised and published field experience summaries), the examples indicate the breadth of ways organisations refer to guidance, and suggest complexities in whether and how humanitarian responses are evidence-based.

Table 4. Examples of guidance-related terms identified in the published field experience summaries 


\begin{tabular}{|c|c|c|c|c|}
\hline Search term(s) & $\begin{array}{l}\text { Number } \\
\text { of exact } \\
\text { matches }\end{array}$ & $\begin{array}{l}\text { Number } \\
\text { with } \\
\text { stemmed } \\
\text { words }\end{array}$ & $\begin{array}{l}\text { Number } \\
\text { of } \\
\text { examples } \\
\text { identified }\end{array}$ & $\begin{array}{l}\text { Selected example of how the search } \\
\text { term was referred to in the published } \\
\text { field experience summaries }\end{array}$ \\
\hline Guidance & 26 & 26 & 13 & $\begin{array}{l}\text { The global nutrition community was still } \\
\text { developing and issuing guidance on } \\
\text { anthropometric measurements. }\end{array}$ \\
\hline $\begin{array}{l}\text { Standard } \\
\text { (Standards) }\end{array}$ & 7 & 14 & 9 & $\begin{array}{l}\text { There is a gap of } 52 \% \text { or } 2,099 \text { beds in } \\
\text { total the number of available hospital } \\
\text { beds compared to global standards. }\end{array}$ \\
\hline Protocol & 20 & 40 & 8 & $\begin{array}{l}\text { The survey team adjusted their } \\
\text { anthropometric-measurement protocol } \\
\text { according to international } \\
\text { recommendations that caution against } \\
\text { height and weight measurements } \\
\text { involving prolonged } \\
\text { contact. }\end{array}$ \\
\hline Guideline & 2 & 11 & 6 & $\begin{array}{l}\text { Each session follows guidelines from } \\
\text { CDP's pre-existing MHPSS services that } \\
\text { have been adapted to COVID- } 19 \text { and the } \\
\text { online format in consultation with } \\
\text { specialised psychologists. }\end{array}$ \\
\hline $\begin{array}{l}\text { Rules } \\
\text { (Rule) }\end{array}$ & 12 & 15 & 5 & $\begin{array}{l}\text { For example, staff and communities still } \\
\text { tend to interact in breach of physical } \\
\text { distancing rules. }\end{array}$ \\
\hline $\begin{array}{l}\text { Procedure } \\
\text { (Procedures) }\end{array}$ & 5 & 19 & 5 & $\begin{array}{l}\text { The COVID-19 standard operating } \\
\text { procedure protocol circulated by the } \\
\text { national government, which was } \\
\text { adapted from the WHO, was used to } \\
\text { develop this training curriculum. }\end{array}$ \\
\hline $\begin{array}{l}\text { Recommendation } \\
\text { (Recommendation, } \\
\text { Recommended) }\end{array}$ & 4 & 12 & 5 & $\begin{array}{l}\text { International Medical Corps did not have } \\
\text { a large stock of Mid-Upper Arm } \\
\text { Circumference tapes prepositioned to } \\
\text { meet the recommendation of using one } \\
\text { tape per household. }\end{array}$ \\
\hline $\begin{array}{l}\text { Measures } \\
\text { (Measure, } \\
\text { Measurement) }\end{array}$ & 98 & 119 & 4 & $\begin{array}{l}\text { All hospital staff underwent a one-week } \\
\text { training on IPC measures in hospitals } \\
\text { and communities. }\end{array}$ \\
\hline $\begin{array}{l}\text { Plan } \\
\text { (Plans, Planned) }\end{array}$ & 39 & 67 & 4 & $\begin{array}{l}\text { Shortly after, the UN Global } \\
\text { Humanitarian Response Plan was } \\
\text { enacted in CAR. The response plan } \\
\text { included awareness-raising sessions, } \\
\text { creation of isolation spaces, } \\
\text { reinforcement of handwashing and } \\
\text { strengthening of epidemiologic } \\
\text { surveillance, although these were slow tc } \\
\text { be implemented. }\end{array}$ \\
\hline Principle & 3 & 5 & 4 & The "no touch" principle was \\
\hline
\end{tabular}



group meetings or home visits.

$\begin{array}{lllll}\begin{array}{l}\text { Requirement } \\ \text { (Requirements, } \\ \text { Required) }\end{array} & 1 & 52 & 4 & \begin{array}{l}\text { Reusable PPE was chosen for three } \\ \text { reasons: }(1) \text { for reasons of space as } \\ \text { reusable PPE takes up less space than } \\ \text { the required stock of disposable PPE. }\end{array} \\ \begin{array}{l}\text { Instruction } \\ \text { Instructed, }\end{array} & 0 & 12 & 4 & \begin{array}{l}\text { Acceptance of instructions to protect } \\ \text { Instructions) }\end{array}\end{array}$

$\begin{array}{lllll}\begin{array}{l}\text { Guide } \\ \text { (Guided, Guiding) }\end{array} & 4 & 39 & 3 & \begin{array}{l}\text { IRC Jordan developed a remote delivery } \\ \text { plan guided by technical documents } \\ \text { from IRC headquarters. }\end{array}\end{array}$

$\begin{array}{llll}\text { Process } & 31 & 42 & 2\end{array}$

At this time, our usual process for inspection of samples and quality checks using certifications, testing, and other processes has been modified to support a distributed workforce, and an evolving list of approved products by the Food and Drug Administration.

$\begin{array}{llll}\text { Advice } & 10 & 2 & \begin{array}{l}\text { Limited coordination between } \\ \text { headquarter, regional and local offices at } \\ \text { the beginning of the response, which led } \\ \text { to inconsistency in the tools, advice and } \\ \text { instructions shared. }\end{array}\end{array}$

$\begin{array}{llll}\text { Evidence } & 8 & 8 & 2\end{array}$

2 This program features a communitybased model and utilises evidencebased approaches to group interpersonal therapy for the refugee community.

$\begin{array}{llll}\text { Information } & 93 & 133 \quad \begin{array}{l}\text { At a time when emergency use } \\ \text { authorisations are implemented and } \\ \text { information about COVID-19 changes } \\ \text { regularly, Americares has dedicated } \\ \text { more manpower to keep pace with the } \\ \text { changing standards. }\end{array}\end{array}$

Form $\quad 16 \quad 23 \quad 1 \quad$ For half a day, a team of two trained
MSF staff, one IPC/logistician and one nurse, evaluate the health structure using a specific assessment form which focuses on IPC, the flow of patients and health workers.

$\begin{array}{llll}\text { Criteria } & 3 & 3 & 1 \\ \text { (Criterion) } & & \end{array}$

Directive

(Directives)
1

39
1

1
Beneficiary triage into risk groups is carried out in alignment with the guidelines and criteria that were approved by the SGBV working group in Lebanon.

Following the MoH's directive for contingency health promotion on COVID- 
Regulation

(Regulations)
1
6
1

ACF adapted their activities to tackle constraints related to the COVID-19 situation, and to best support and protect staff and beneficiaries, following government and Inter Sector

Coordination Group (ISCG) regulation.

Total

384

695

85

21

\section{Discussion}

The literature on evidence-based humanitarian intervention argues that humanitarian response and programming decisions should take into account data and information derived from reliable assessments of needs, and evaluations of intervention effectiveness [14,15]. However, there is also pragmatic recognition that experience-based response and expert judgment is ubiquitous in humanitarian settings, given the challenges of timely and reliable data collection and analysis in crisis contexts to generate adequate evidence [16]. Combining these approaches, Darcy et al. "understand a well-informed decision to be one that takes due account of data and information most relevant to the crisis context, and combines this with experience-based knowledge to determine what intervention is the most appropriate in that context" [17]. Yet, despite recognising that humanitarian response should be evidence-based, it is widely lamented that many interventions are not [15,18-20].

\section{Key factors for implementing evidence-based response}

Our study suggests that determining whether humanitarian interventions and adaptations are evidencebased, experienced-based, or grounded in a mix of information and intuition, may be more complex than previously assumed. Our analysis of humanitarian organisation's field experiences when responding and adapting to COVID-19 identified four factors relevant to the use of evidence-based guidance, and the nature of that usage: availability and access; coherence and coordination; contextual relevance; trust and credibility. We argue these factors may have an important influence on the extent to which humanitarian response is or can be based on evidence, particularly in dynamic and uncertain humanitarian and fragile settings affected by the COVID-19 pandemic.

\section{Availability and access}

As depicted in Table 3, global guidance documents were available on a wide range of framework areas, and our interviews only identified a few specific gaps in terms of topic coverage. For example, one organisation lacked guidance for programming with mobile populations that was specific enough to the 
complexities of migration journeys in COVID-19 affected settings. Another found that guidance on the modalities and timing for resuming activities and transport post-lockdown was missing. Initially, our guidance document review found some framework areas to be under-documented, such as 'sexual and reproductive health', 'human resources' and 'coordination'. However, on closer analysis, these topics were often included within documents categorised under other framework areas. For instance, guidance on adapting existing food security and livelihoods interventions to COVID-19 often includes a section on the safety of staff during food distribution activities, and a section on how to coordinate with other stakeholders. Guidance on maternal, newborn and child health often includes a section on sexual and reproductive health. Thus, guidance on most framework areas was available, even if some topics were more difficult to access by being bound up among broader topics. Clearly, availability of evidence-based guidance is a key first step in order for humanitarian organisations to base their implementations on it. Alone, however, availability is insufficient: access is also crucial.

We identified the importance of access from our analysis of available guidance by organisation. Although we found guidance was available from a wide range of organisations (see Table 1), we identified some limitations in the way large INGOs disseminate guidance, which may create access challenges for less resourced organisations. During the initial review period (March to May 2020), we could not identify guidance documents from major INGOs including MSF, Save the Children, and BRAC, as they did not publish them openly on their organisational websites nor on general humanitarian resource websites (such as Reliefweb or humanitarian cluster websites). We later collected guidance documents from those organisations directly after interviews with their staff or following a more detailed investigation and personal follow-up. We learned that although these organisations had developed various COVID-19 guidance, they had initially kept these documents internal.

We find this practice problematic: by making their guidance more readily available, large INGOs could facilitate more evidence-based programming for other organisations. INGOs specialised in humanitarian response in particular are well placed to disseminate the types of guidance recommended for humanitarian and fragile settings, such as context-specific frameworks [21] and resource-stratified guidelines [22]. They also play an important role in disseminating global standards to local levels, as was evident in the way that many larger INGOs conducted remote in-house trainings based on guidance derived from global WHO standards and adapted to context. Extending such trainings to a wider range of local partners could substantially improve availability and access to evidence-based guidance. For sensitive or private operations, approaches that allow for guidance to be segmented while allowing the main content to be disseminated publicly should be explored. Mechanisms to systematically disseminate new and revised guidance to ground level should also be improved to allow for more fluid and effective adaptation and response.

\section{Coherence and coordination}

While making additional guidance available and accessible can be fruitful, striking a coherent balance of quantity and quality, breadth and specificity, and the format of dissemination, is also important. On the 
one hand, our interviews revealed cases where the guidance available was too general or designed for a broad public audience, rather than tailored to the health and humanitarian professionals who actually implement humanitarian programmes. On the other hand, some organisations found FAQs, information sheets, key messages and other communications materials (as opposed to detailed technical guidance documents) helpful when creating localised response plans and procedures.

These contrasting experiences suggest the utility of technical and non-technical information varies by actor and context. Indeed, our results showed many cases of organisations collating global guidance with local, national, regional materials, as well as mixing guidance of various formats together. Such practices illustrate the importance of a having wide range of guidance messages and formats available, from both global and local levels, to ensure the uptake of evidence in humanitarian and health responses. However, they also place an onus on guidance setters to better distinguish between public information campaigns, and technical guidance for health and other humanitarian programmes, to ensure coherence between these different types of documents and formats. For example, public information tailored to combat the infodemic may not be optimal for sensitising and educating humanitarian and health specialists, who require detailed information tailored to their purposes. Conversely, highly technical procedures may not be fit for purpose in all fragile operational settings, as Hussain \& Sen found in their analysis of overly bureaucratic guidance issued by the European Union for managing COVID-19 in Syria [23].

In addition, multiple interview respondents mentioned challenging discrepancies between different COVID-19 guidance documents, for example, between those disseminated by global (UN) organisations, other humanitarian organisations, and national $(\mathrm{MoH})$ institutions. This variation made it difficult for humanitarian organisations to decide which guidance to follow. In CAR, MSF described efforts to align guidelines with local partner organisations as a fragmented, decentralised process. In other cases, the rapidly emerging and evolving evidence base meant that guidance was also rapidly changing, which posed challenges not only in the design and implementation of interventions, but also in gaining community trust and maintaining credibility. This finding suggests continued need for improved information management and coordination, at all levels of humanitarian response, which has long been recognised [24]. The flurry of information service efforts that emerged as large workforces rapidly found themselves desk-bound, while well intentioned, also generated duplication and confusion. Our own COVID-19 Humanitarian platform project experienced this, when we found an individual website had created similar online compilation of guidance documents, prompting us to reach out and coordinate efforts. Thus, in addition to coherence, there is an important role for coordination to ensure guidance is updated, aligned, contextually grounded and responsive to the evolving situation.

\section{Contextual relevance}

Our study also found it is important for guidance to be appropriate and sensitive to context, in terms of language, information requirements, and technological realities. Previous recommendations have stressed that guidance on how to respond to humanitarian crises should consider data demonstrating 
priority needs, efficacy and cost-effectiveness in each context [18]. The fact that most humanitarian organisations we interviewed had to adapt guidance to context suggests humanitarian guidance setters are yet to meet these recommendations.

Multiple organisations explained how global guidance needed to be translated and adapted to their local language, which took time and often required expert consultation. Country-level coordination clusters, such as the WASH cluster, developed national guidance notes that are publicly available. However, the scarcity of global guidance directly written in a variety of languages and local dialects has been found to limit access to information in COVID-19 and other crises [25-28]. The need for translation applies not only to new COVID-19 specific documents, but also to evidence reviews from past epidemics such as Cholera and Ebola, which remain published mainly in English. As many countries currently facing humanitarian crises are French, Spanish or Arabic-speaking countries, the lack of evidence in these languages is particularly problematic. The Africa CDC systematically translates its guidance into French, Arabic, and Portuguese, which is good practice more organisations and institutions could emulate. However, even with major languages covered, the need for translation to the hundreds of languages used at the operational level requires more innovative solutions, including capitalising on automated translation and transcription technologies and natural language processing tools.

We also found that the local data and information landscape has an important relationship with the appropriateness of guidance. REACH encountered multiple challenges to design a severity index that was sensitive to local complexities and flexible enough to handle dynamic and uncertain data generation processes. For instance, they had to refine the general list of indicators for severity to a more limited set when they examined the (lack of) locally available data at the level of the 'zone de santé', or health zone. Moreover, they had to retain enough room in the model to add indicators later as more data would become available, and weight for differences in the quality of data across indicators and zones. They also had to take into consideration the secondary impacts of COVID-19, such as on food markets and employment, which were as relevant to severity as the direct impacts on health systems and outcomes. Thus, while methods to quantify and compare severity at the global level continue to advance, it is important that they remain cognisant that many globally conceived models may not generalise to complex local humanitarian settings.

Matching recommended COVID-19 responses as outlined in guidance documents with contextually appropriate technology and resources was another area where organisations faced challenges, and innovated appropriate solutions. For example, guidance on social media messaging for risk communication that assumes internet connectivity or personal device access may have little relevance for contexts where blanket radio or megaphone broadcasting remain prominent. In multiple contexts studied, including Uganda and South Sudan, megaphone broadcasting was found to be an effective risk communication modality. In Nepal, a key enabling factor for a local organisation who adapted their education programmes to an online format was the access of one village elder to a smart phone and data package, which students then borrowed to watch educational videos. In Libya, one organisation placed QR codes in the market with links to a smartphone survey, so they could monitor prices without 
having enumerators physically present. These examples point to wide variation in technology levels by context, which have implications for guidance setting and dissemination, and correspondingly the uptake of this evidence for response.

\section{Trust and credibility}

Finally, trust played an important role in relation to guidance use, particularly in terms of effectively implementing evidence-based response strategies. Previous studies have found successful implementation of guidance protocols requires securing the trust and confidence from both the target community and the regulatory authorities. A rapid evidence review of community engagement for infection prevention and control during previous epidemics, for example, identified trust as a key success factor [29].

Trust in humanitarian organisations in the COVID-19 context has been further complicated in two directions. Widespread rumours and misinformation have engendered competing narratives and alternative solutions to COVID-19, which erode community trust in humanitarian organisations trying to implement guidance-based responses. In addition, humanitarian organisations also faced mistrust from host governments and local authorities, who often needed to be convinced before guidance could be disseminated and programmes implemented. In refugee camps on Lesbos, Greece, MSF described that guidance to isolate vulnerable persons from the broader camp population was not an approach supported by local authorities, hence alternative approaches had to be discussed before evidence-based programmes could be implemented.

Humanitarian organisations reported using various strategies to gain the trust needed to implement their responses and adaptations. For example, communication with local authorities including the police force was helpful in many contexts. Partnerships, in particular, collaboration with the $\mathrm{MoH}, \mathrm{UN}$ organisations, other INGOs and NGOs, were also integral for organisations to build space and confidence to introduce new or adapt existing interventions. Engagement with the national $\mathrm{MoH}$ was particularly important for a wide range of tasks: gaining permission to access sensitive areas, using national hotlines, surveillance and reporting, securing additional supplies, receiving weekly updates, building strategies and action plans, or deploying staff to/from the government response. At times, organisations reporting good relationships with the local health authorities also experienced success in gaining trust of the local population, though this may be contingent on the community's own relationship with authorities. In general, whether trust of the community, government, or both were required in a given context, successful implementation of guidance protocols was heavily dependent on securing the requisite trust.

\section{Implications for evidence-based humanitarian response}

Guidance, when considered broadly played an important role in the design and implementation of responses and adaptations to COVID-19. The wide range of terms organisations use to refer to guidance show that they are concerned with applying various standards and protocols, or at least comparing their 
innovated responses with existing benchmarks and documents. This suggests that evidence-based humanitarian response may be more prevalent than previously expected.

At the same time, taking a wide view of guidance identifies a diverse set of challenges associated with designing, disseminating and implementing evidence-based humanitarian response. Humanitarian organisations' tendency to compile and adapt various guidance to steer their COVID-19 responses in a way that was suitable for their contexts indicates that global guidance is not entirely relevant to address programmatic challenges at local level. Unfortunately, organisations at the local level often lack the time or resources to develop their own guidance de novo. Moreover, the exact mix of guidance and innovation driven response varies widely depending on the organisation and context in question. Thus, to be relevant and applied, guidance should take into consideration the ground-level context, resources and language, and be responsive to actual patterns in the use of already available guidance. Considering availability of and access to guidance, coherence and coordination, contextual relevance, and trust and credibility may help foster the implementation of more evidence-based response.

As both the global pandemic and humanitarian contexts are highly dynamic, it is also important that these factors are considered in a dynamic and responsive way. The COVID-19 Humanitarian platform provides a potential model for a more circular, dynamic and responsive evidence-based guidance and implementation process. By combining guidance documents with detailed experiences and anecdotes from the field, documented via qualitative case studies, surveys, webinars, and discussion boards, the platform offers users the chance to consult what is recommended alongside what is actually happening on the ground. In particular, the precise description of the modalities and rational for innovating, adjusting and implementing programmes can be extracted in real-time to provide a resource for global standard setters to consider when developing guidance, particularly in emergency humanitarian response and fastpaced epidemics.

Such a feedback loop could complement the prevailing guidance development model, which relies on updating existing guidance from previous epidemics, and which may not necessarily be relevant to the specific contexts and challenges of contemporary crises. It may also help to counter information asymmetries, such as those experienced in the early phases of COVID-19: transmission patterns from East to West/North before reaching the South led to a proliferation guidance designed for advanced economies, with limited relevance for low-income and humanitarian crisis settings. Incorporating documented qualitative experiences from these settings could therefore help to generate genuinely global guidance. Other studies have found feedback loops are important for circular guidance use, generation, and adaptation [30,31], while academic-humanitarian partnerships [32] have also been useful in such endeavours. While the COVID-19 Humanitarian Platform has room to improve, it could serve as basis for more dynamic and responsive evidence implementation in future.

\section{Limitations}

To our knowledge, this is the first study investigating the use of COVID-19 guidance in humanitarian settings. It covers a wide range of humanitarian settings and organisations, and the in-depth interviews 
reveal details on multiple aspects of response and adaptation to COVID-19, including guidance and evidence use. However, few interviews focused explicitly or solely on the use of guidance. Rather, in explaining the detailed modalities and rationales behind their interventions and adaptations, organisations made natural references to various global and national level guidance documents, as well as internal guidelines, protocols or procedures. A strength of this approach is that we avoided priming respondents to mention guidance which would otherwise have been omitted. A weakness is that we may have missed further different ways in which organisations used and referred to guidance.

Another limitation of the study design is the risk of selection bias among the organisations interviewed. Although we attempted to ensure diversity of interview participants by tracking location and type of organisation, humanitarian organisations may have self-selected into the study, for example by completing the online form to promote their interventions or because they had sufficient time or resources to complete the form. In addition, the use of a convenience approach to sampling and snowballing participants from our own connections meant the coverage of contexts likely favours locations and contexts where the research team had previously worked or conducted research, and may omit other important humanitarian contexts. Nonetheless, we remain confident our sample reflects an informatively diverse range of organisations and humanitarian contexts for our qualitative approach.

\section{Conclusion}

This study aimed to analyse the relevance and use of global humanitarian guidance according to documented humanitarian field experiences of COVID-19 responses and adaptations. Experiences from the various crisis settings indicated that adopting guidance to respond and adapt to COVID-19 is not a linear process, but rather a complex interplay involving substantial innovation and adaptation, to meet the demands and constraints of the local context. We found that organisations refer to guidance and evidence-based processes in myriad ways, and use guidance in three main ways: adopting global guidance, using guidance disseminated from within their organisation, or combining multiple sources of guidance. However, in most cases, organisations had to adapt this guidance to context. This adaptive process revealed four key factors that were important to the implementation of an evidence-based humanitarian response: access and availability, coherence and coordination, contextual relevance, and trust and credibility. Our findings imply that the development of guidance aimed at humanitarian actors could be enhanced through responsive incorporation of contextualised field experiences in a timely manner, using real-time feedback loops that link global evidence-based guidance with ground-level realities. The COVID-19 Humanitarian platform offers a model for this more nuanced and response vision of evidence and experience-based response.

\section{List Of Abbreviations}

ALNAP: $\quad$ Active Learning Network for Accountability and Performance in Humanitarian Action CAR: $\quad$ Central African Republic 
CDC: $\quad$ Centers for Disease Control and Prevention

CDP: $\quad$ Centre for Disaster Preparedness

DRC: Democratic Republic of the Congo

IASC: Inter Agency Standing Committee

IFRC: International Federation of Red Cross

INGO: International Non-Governmental Organisation

IPC: Infection Prevention and Control

IRC: International Rescue Committee

LRCS: $\quad$ Libyan Red Crescent Society

LSHTM: London School of Hygiene and Tropical Medicine

MHPSS: Mental Health and Psychosocial Support

MoH: $\quad$ Ministry of Health

MSF: $\quad$ Médecins Sans Frontières

NGO: Non-Governmental Organisation

NICE: National Institute for Health and Care Excellence

UN OCHA: United Nations Office for the Coordination of Humanitarian Affairs

PPE: $\quad$ Personal Protective Equipment

RCCE: Risk Communication and Community Engagement

SGBV: $\quad$ Sexual and Gender-Based Violence

UN: $\quad$ United Nations

UNICEF: United Nations Children's Fund

WASH: Water, Sanitation and Hygiene

WHO: $\quad$ World Health Organization

\section{Declarations}




\section{Ethics approval and consent to participate}

The three universities coordinating the COVID-19 Humanitarian platform (Center for Humanitarian Health (Johns Hopkins University), the Health in Humanitarian Crises Centre (London School of Hygiene and Tropical Medicine), and the Geneva Centre of Humanitarian Studies (University of Geneva, The Graduate Institute) secured ethical approval for the as a study of non-human subjects (organisations) at the outset of the project in March 2020.

\section{Consent for publication}

We provided each participant with information on the project prior to the interview, and requested informed consent to record the interview, display the organisation's name and location on the platform, upload the summarised information to COVID-19 Humanitarian platform, and use the information for other analysis (such as this paper). Participants granted verbal consent to record the interviews in most cases; we wrote detailed notes if no recording or transcript was possible. We recorded individual participant names for follow-up contact purposes but did not display these publicly. Some organisations requested anonymity.

\section{Availability of data and materials}

The COVID-19 Humanitarian platform (covid19humanitarian.com) offers free access to all the published field experiences and guidance documents collected. Further datasets generated and analysed may be provided on request.

\section{Competing interests}

The authors declare no competing interests.

\section{Funding}

This study was funded by the Geneva Centre of Humanitarian Studies.

\section{Authors' contributions}

The study was conceptualised by $A O$ and $K B$. KB and $A M$ conducted the guidance document analysis. RJ and $A O$ conducted the field interview analysis. $A O$ and RJ drafted the paper. PS, CA, NSS, KB and AM provided feedback and edits. All authors read and approved the final manuscript.

\section{Acknowledgements}

We extend our sincerest gratitude to the humanitarian organisations and interview participants for sharing their valuable time and experiences. We thank our entire COVID-19 Humanitarian platform team of volunteer researchers across the three partner universities: Danielle Bancroft, Kim Berg, Anne Bergman, Mariana Nogueira De Abreu Pinto Rodo, Michael Diaz, Lisa Diemiger, Ahmed El Refai, Alix Faddoul, 
Caroline Favas, Alexandra Molina Garcia, Alexandra Humpert, Ada Humphrey, Sharif Ismail, Hanad Karie,

Zélie Kasten, Samantha Law, Aaron Littlefield, Ruth Lorimer, Masanja Saimon Lumaneja, Wincate Maingi, Emily Meyer, Céline de Richoufftz, Jacqueline Safieh, Martha Teshome, Nima Yaghmaei. We also thank Lisa Diemiger for additional proof reading of the manuscript.

\section{Authors' information}

Alex Odlum, Geneva Centre of Humanitarian Studies, University of Geneva and the

Graduate Institute, Switzerland

Rosemary James, Geneva Centre of Humanitarian Studies, University of Geneva and the

Graduate Institute, Switzerland

Audrey Mahieu, Geneva Centre of Humanitarian Studies, University of Geneva and the

Graduate Institute, Switzerland

Karl Blanchet, Geneva Centre of Humanitarian Studies, University of Geneva and the

Graduate Institute, Switzerland

Chiara Altare, Center for Humanitarian Health, Johns Hopkins Bloomberg School of

Public Health, USA

Neha S Singh, Health in Humanitarian Crises Centre, London School of Hygiene and

Tropical Medicine, UK

Paul B Spiegel, Center for Humanitarian Health, Johns Hopkins Bloomberg School of

Public Health, USA

\section{References}

1. UN OCHA. Global Humanitarian Overview 2020. Geneva; 2019.

2. Banatvala N, Zwi AB, Holzer A, Kocher B, Bendahan S, Vonèche Cardia I, et al. Public health and humanitarian interventions: developing the evidence base. BMJ. British Medical Journal Publishing Group; 2000;321:3675.

3. Smith J, Roberts B, Knight A, Gosselin R, Blanchet K. A systematic literature review of the quality of evidence for injury and rehabilitation interventions in humanitarian crises. International Journal of Public Health. 2015;60. 
4. Blanchet K, Ramesh A, Frison S, Warren E, Hossain M, Smith J, et al. Evidence on public health interventions in humanitarian crises. The Lancet. Elsevier; 2017;390:2287-96.

5. UHC2030 Fragile Settings Technical Working Group. COVID-19 and fragile settings. 2020.

6. Singh NS, Abrahim O, Altare C, Blanchet K, Favas C, Odlum A, et al. COVID-19 in humanitarian settings: documenting and sharing context-specific programmatic experiences. Conflict and Health. BioMed Central; 2020;14:79.

7. UN OCHA. COVID-19 Global Humanitarian Response Plan. 2020.

8. UNHCR. Refugee Data Finder [Internet]. 2020 [cited 2021 Apr 30]. Available from: https://www.unhcr.org/refugee-statistics/

9. Tong A, Sainsbury P, Craig J. Consolidated criteria for reporting qualitative research (COREQ): A 32-item checklist for interviews and focus groups. International Journal for Quality in Health Care. 2007;19:34957.

10. Singh NS, Abrahim O, Altare C, Blanchet K, Favas C, Odlum A, et al. COVID-19 in humanitarian settings: documenting and sharing context-specific programmatic experiences. Conflict and Health. BioMed Central; 2020;14:79.

11. Favas C. Guidance for the prevention of COVID-19 infections among high-risk individuals in camps and camp-like settings. London; 2020.

12. European Commission: DRMKC - Disaster Risk Management Knowledge Centre. INFORM Severity [Internet]. 2020 [cited 2021 Apr 30]. Available from: https://drmkc.jrc.ec.europa.eu/inform-index/INFORMSeverity

13. International Rescue Committee. COVID-19 Operational Guidance Note: Mental Health and Psychosocial Support (MHPSS) within Health Programs. 2020.

14. Kayabu B, Clarke M. The use of systematic reviews and other research evidence in disasters and related areas: Preliminary report of a needs assessment survey. PLoS Currents. Public Library of Science; 2013;1-10.

15. Jillson IA, Clarke M, Allen C, Waller S, Koehlmoos T, Mumford W, et al. Improving the science and evidence base of disaster response: A policy research study. BMC Health Services Research. BioMed Central Ltd.; 2019;19:274.

16. Smith J, Blanchet K. Research Methodologies in Humanitarian Crises [Internet]. 2019. Available from: https://www.elrha.org/wp-content/uploads/2020/02/R2HC-Research-Methodologies-in-HumanitarianCrises-new.pdf 
17. Darcy J, Stobaugh H, Walker P, Maxwell D. The Use of Evidence in Humanitarian Decision Making. 2013.

18. Spiegel PB. The humanitarian system is not just broke, but broken: recommendations for future humanitarian action. The Lancet. Elsevier; 2017;

19. Kohrt BA, Mistry AS, Anand N, Beecroft B, Nuwayhid I. Health research in humanitarian crises: an urgent global imperative. BMJ Global Health. 2019;4:1870.

20. Smith J, Blanchet K, Frison S. Does the Humanitarian Sector Use Evidence-informed Standards? A Review of the 2011 Sphere Indicators for Wash, Food Security and Nutrition, and Health Action. PLoS Currents [Internet]. 2018;1-23. Available from: https://currents.plos.org/disasters/?p=38647

21. Gaffey MF, Waldman RJ, Blanchet K, Amsalu R, Capobianco E, Ho LS, et al. Women's and Children's Health in Conflict Settings 4 Delivering health and nutrition interventions for women and children in different conflict contexts: a framework for decision making on what, when, and how. The Lancet. 2021;397:543-54.

22. Alawa J, Alawa N, Coutts A, Sullivan R, Khoshnood K, Fouad FM. Addressing COVID-19 in humanitarian settings: A call to action. Conflict and Health. BioMed Central Ltd; 2020. p. 64.

23. Hussain HY, Sen K. EU guidance impedes humanitarian action to prevent COVID-19 in Syria. The Lancet Global Health. Elsevier; 2020;8:e1112-3.

24. Ramalingam B. Tools for Knowledge and Learning: A Guide for Development and Humanitarian Organisations. London; 2006.

25. O'Brien S, Federici FM. Crisis Translation: Considering Language Needs in Multilingual. Disaster Prevention and Management: An International Journal. 2019;

26. Piller I, Zhang J, Li J. Linguistic diversity in a time of crisis: Language challenges of the COVID-19 pandemic. Multilingua. Walter de Gruyter GmbH; 2020;39:503-15.

27. O’Brien S, Federici F, Cadwell P, Marlowe J, Gerber B. Language translation during disaster: A comparative analysis of five national approaches. International Journal of Disaster Risk Reduction. 2018;31:627-36.

28. Federici FM, Gerber BJ, O’Brien S, Cadwell P. The International Humanitarian Sector and Language Translation in Crisis Situations: Assessment of Current Practices and Future Needs. London; Dublin; Phoenix; 2019.

29. Gilmore B, Ndejjo R, Tchetchia A, De Claro V, Mago E, Diallo AA, et al. Community engagement for COVID-19 prevention and control: A rapid evidence synthesis. BMJ Global Health. BMJ Publishing Group; $2020 ; 5$. 
30. Holzer A, Kocher B, Bendahan S, Vonèche Cardia I, Mazuze J, Gillet D. Gamifying knowledge sharing in humanitarian organisations: a design science journey. European Journal of Information Systems. Taylor \& Francis; 2020;29:153-71.

31. Bonino F, Jean I, Clarke PK. Humanitarian feedback mechanisms: research, evidence and guidance. London; 2014.

32. Aluisio AR, Zhu E, Gil G, Kenyon T, Uzevski V, Levine AC. Academic-humanitarian partnerships: leveraging strengths to combat COVID-19. Global Health Action. Taylor \& Francis; 2020;13:1797296.

\section{Figures}

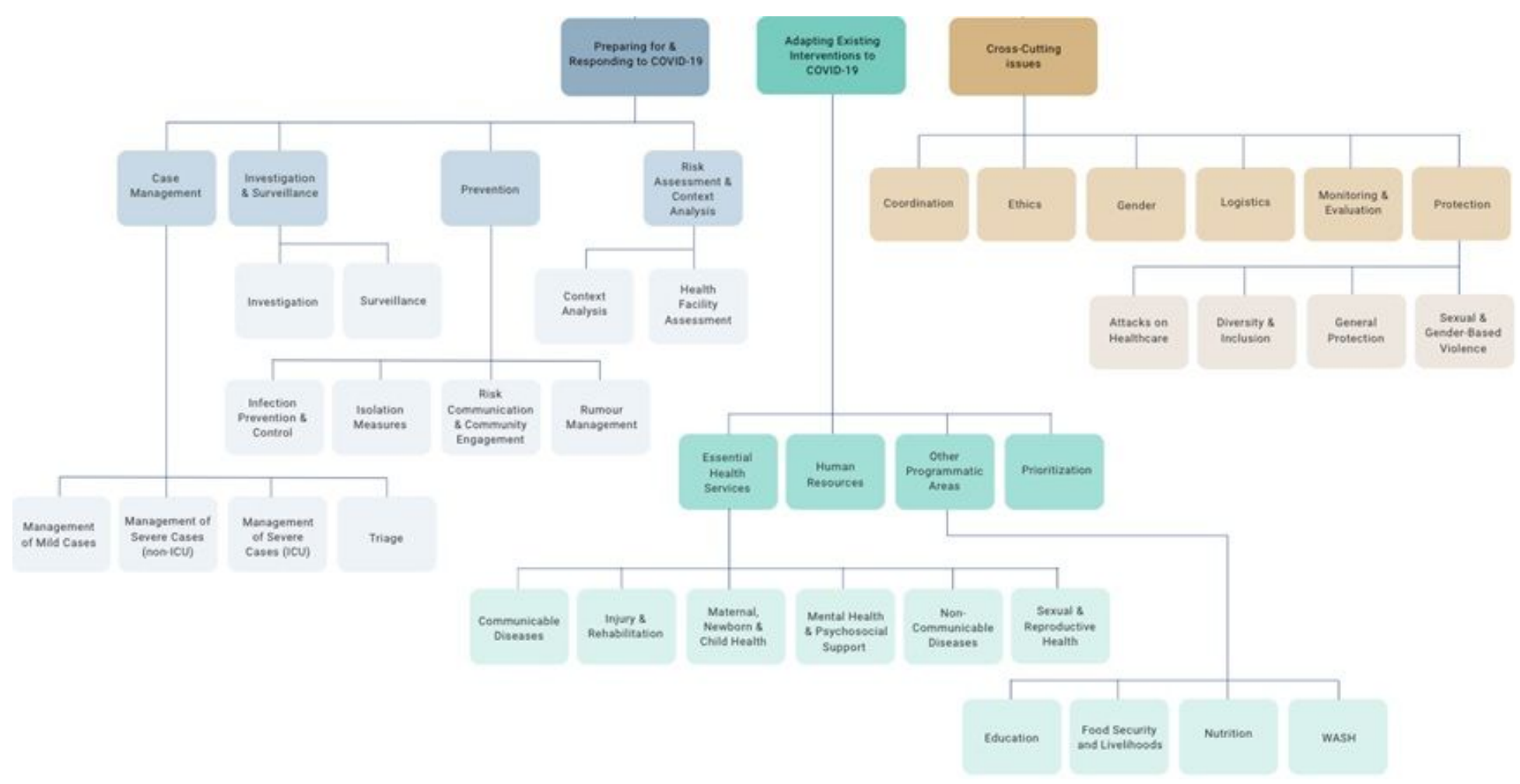

Figure 1

COVID-19 Humanitarian Platform - Framework for guidance and field experience collection 